\title{
Surfactant-Mediated Lithium Orthosilicate Composite Enables Rapid High-Temperature
}

$\mathrm{CO}_{2}$ Absorption

Anthony Vallace ${ }^{1}$, Simon Brooks ${ }^{1}$, Joseph Mendez ${ }^{1}$, Rocco D’Ascanio ${ }^{1}$, Michael A. Smith ${ }^{1 *}$, Charles $\mathrm{Coe}^{1}$

${ }^{1}$ Department of Chemical Engineering, Villanova University, Villanova, PA 19085 (USA)

*michael.a.smith@,villanova.edu 
Using ethanol as a co-solvent affects the initial rate of adsorption:

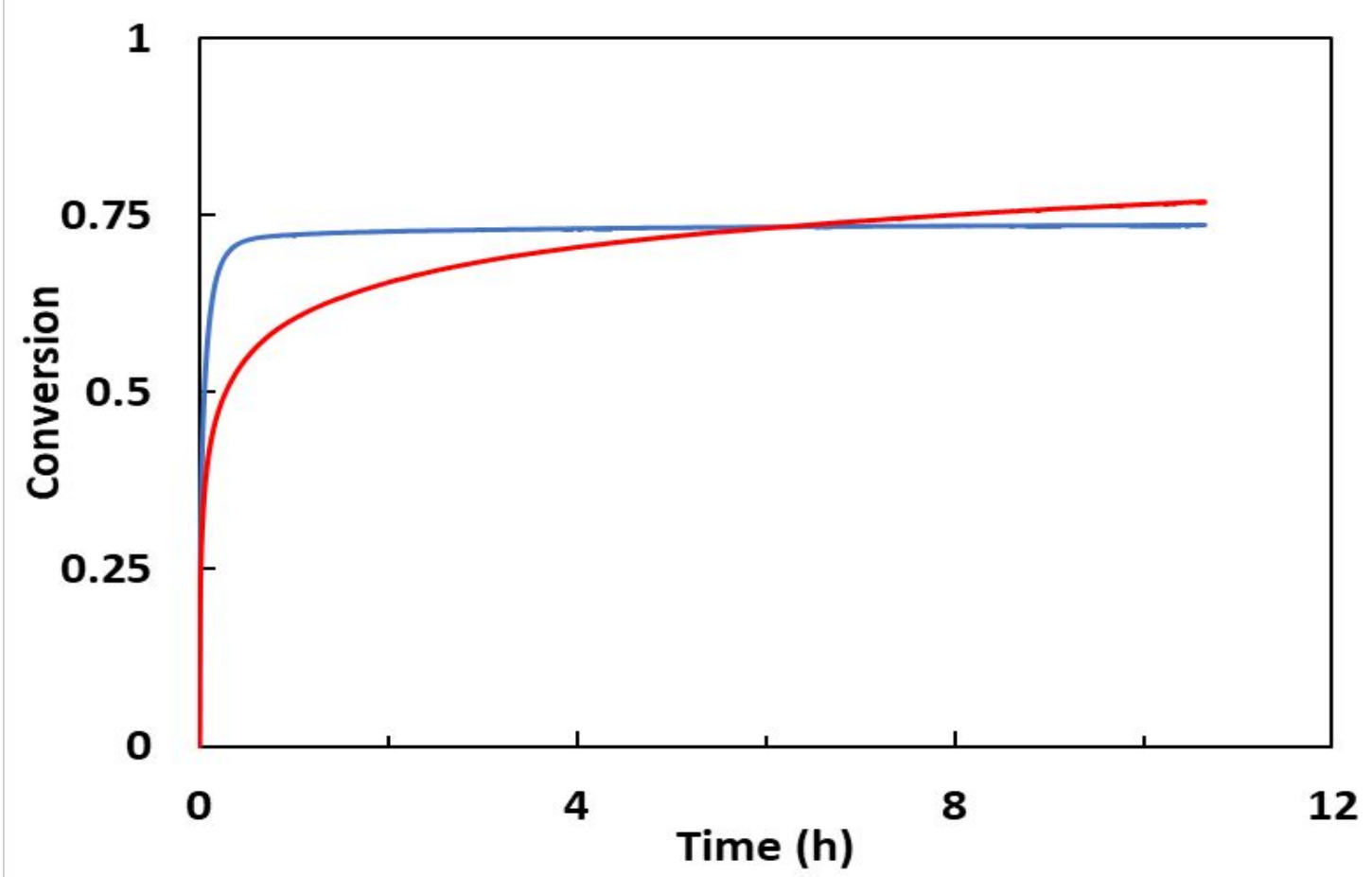

Figure S1. TGA uptake curves at $923 \mathrm{~K}$ and $50 \mathrm{vol} \% \mathrm{CO}_{2} / \mathrm{N}_{2}$ for $\mathrm{SM}(0.06)$ with (blue) and without (red) ethanol.

\section{Variable $\mathrm{CO}_{2}$ Concentration studies:}

The equilibrium $\mathrm{CO}_{2}$ loading was explored across different $\mathrm{CO}_{2}$ concentrations. Figures $\mathrm{S} 2$ and $\mathrm{S} 3$ show the $\mathrm{CO}_{2}$ uptake curves for $\mathrm{SM}(0.12)$ in $10 \%, 25 \%, 50 \%$, and $75 \% \mathrm{CO}_{2}$ at 873 and $923 \mathrm{~K}$, respectively. Table $\mathrm{S} 1$ shows the initial reaction rates at 873 and $923 \mathrm{~K}$ under different $\mathrm{CO}_{2}$ concentrations. 


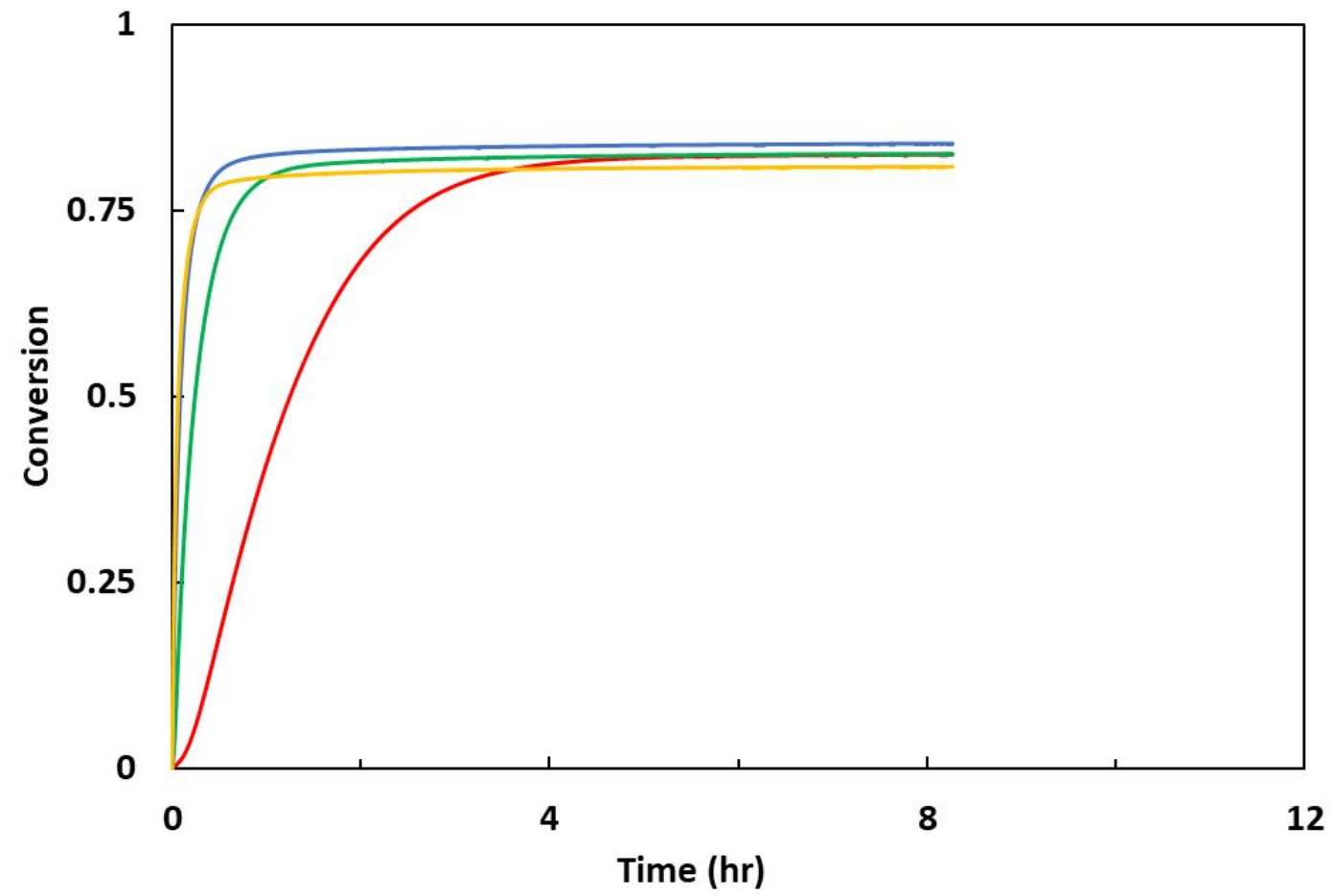

Figure S2. $\mathrm{CO}_{2}$ uptake curves at $873 \mathrm{~K}$ for $\mathrm{SM}(0.12)$ (at 10 (red), 25 (green), 50 (blue) and 75 (yellow) vol\% $\mathrm{CO}_{2}$.

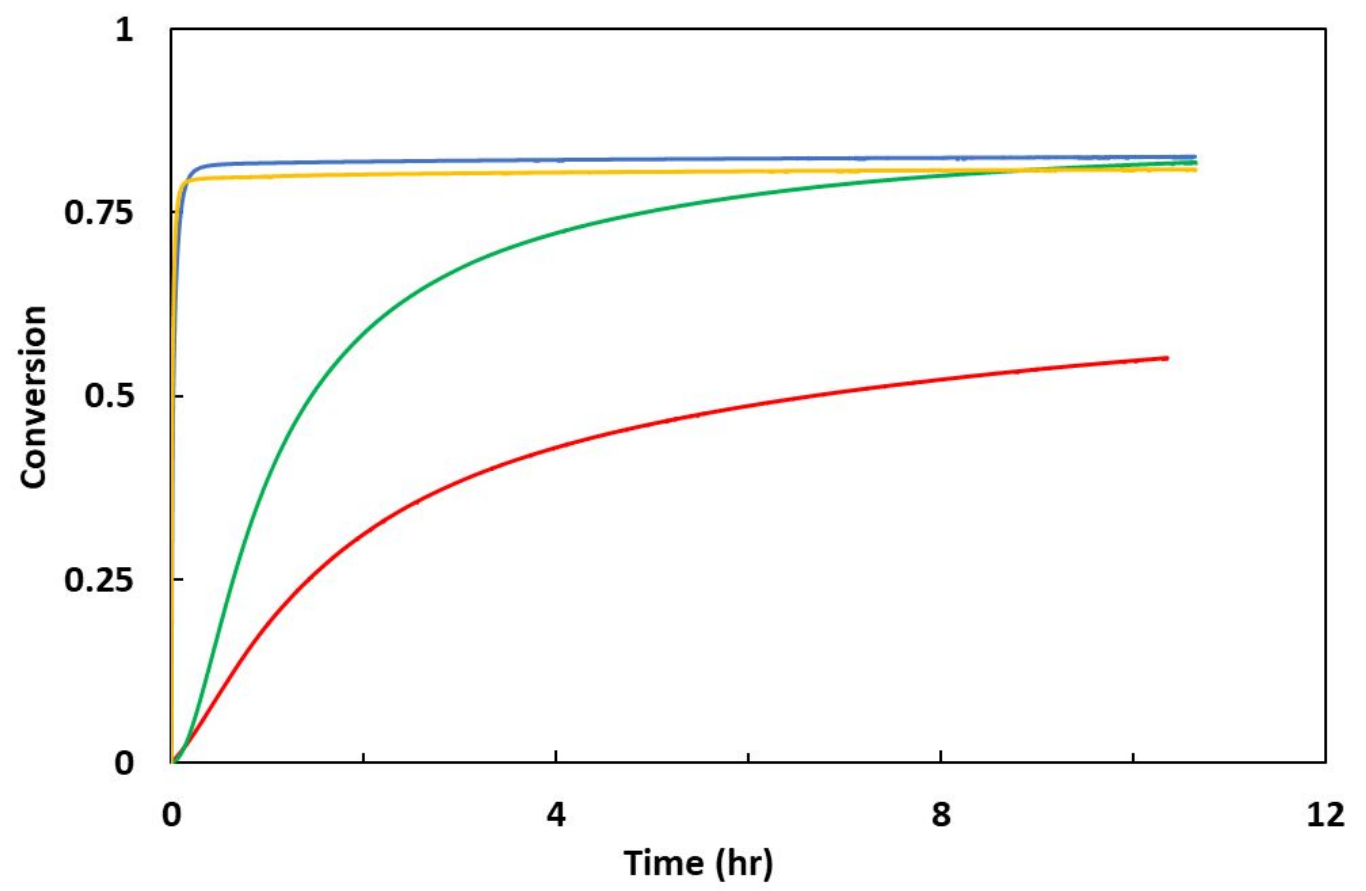

Figure S3. $\mathrm{CO}_{2}$ uptake curves at $923 \mathrm{~K}$ for $\mathrm{SM}(0.12)$ at $10,25,50$ and $75 \mathrm{vol} \% \mathrm{CO}_{2} / \mathrm{N}_{2}$. 
Table S1. Initial reaction rates for $\mathrm{SM}(0.12)$ in different $\mathrm{CO}_{2}$ concentrations at 873 and $923 \mathrm{~K}$.

\begin{tabular}{ccc}
\hline $\begin{array}{c}\text { Concentration } \\
\text { (vol\%) }\end{array}$ & $\begin{array}{c}\text { Initial Reaction Rate }\left(\mathbf{w t} \% \mathbf{C O}_{\mathbf{2}} \mathbf{~ m i n}^{-1}\right) \\
\mathbf{8 7 3 ~ K}\end{array}$ & $\mathbf{9 2 3} \mathbf{~ K}$ \\
\hline \hline 10 & 0.3 & 0.12 \\
25 & 1.9 & 0.3 \\
50 & 6.0 & 19 \\
75 & 9.0 & 44 \\
\hline
\end{tabular}

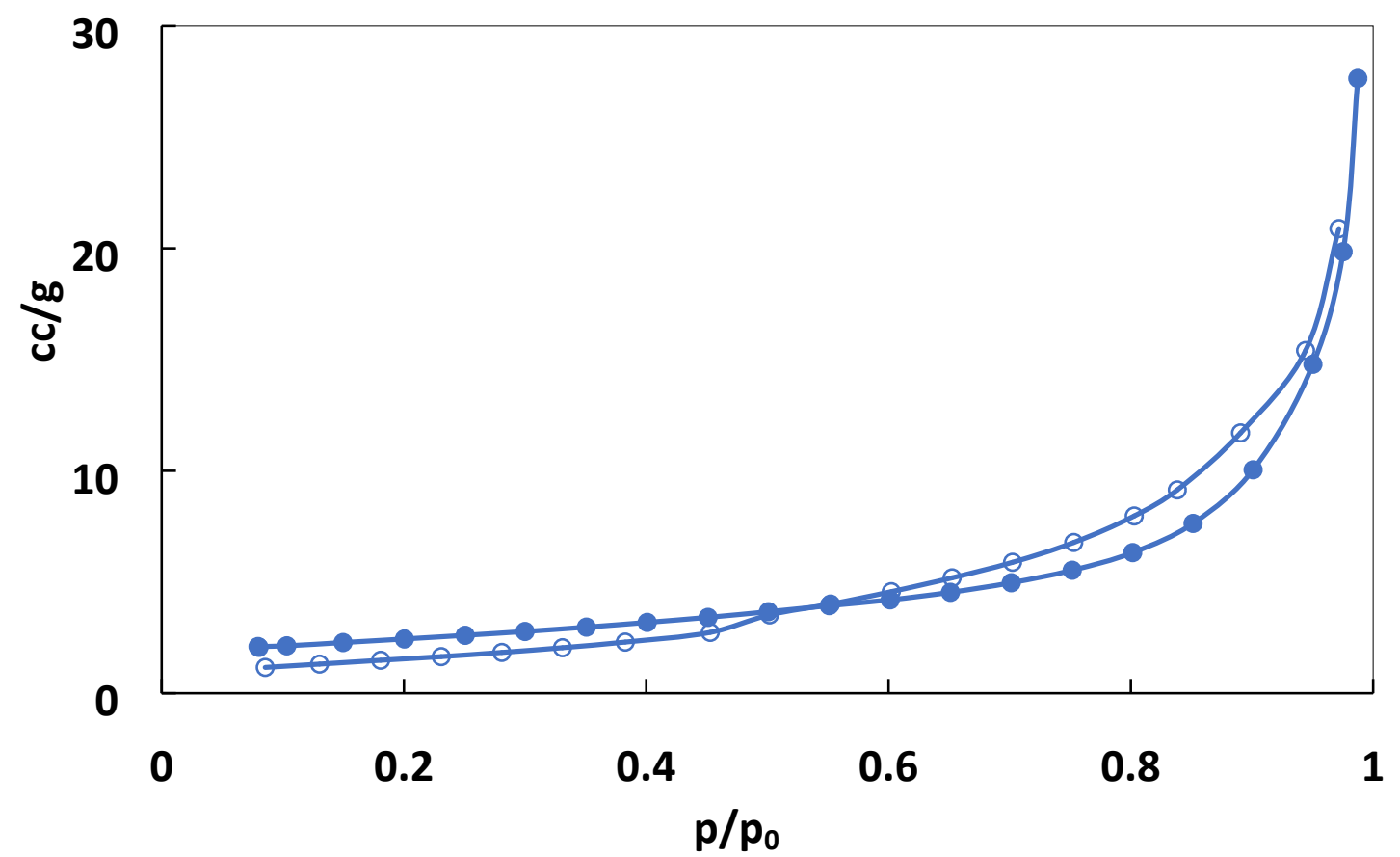

Figure S4. $\mathrm{N}_{2}$ isotherm at $77 \mathrm{~K}$ for $\mathrm{SM}(0.12)$; open circles are desorption. 


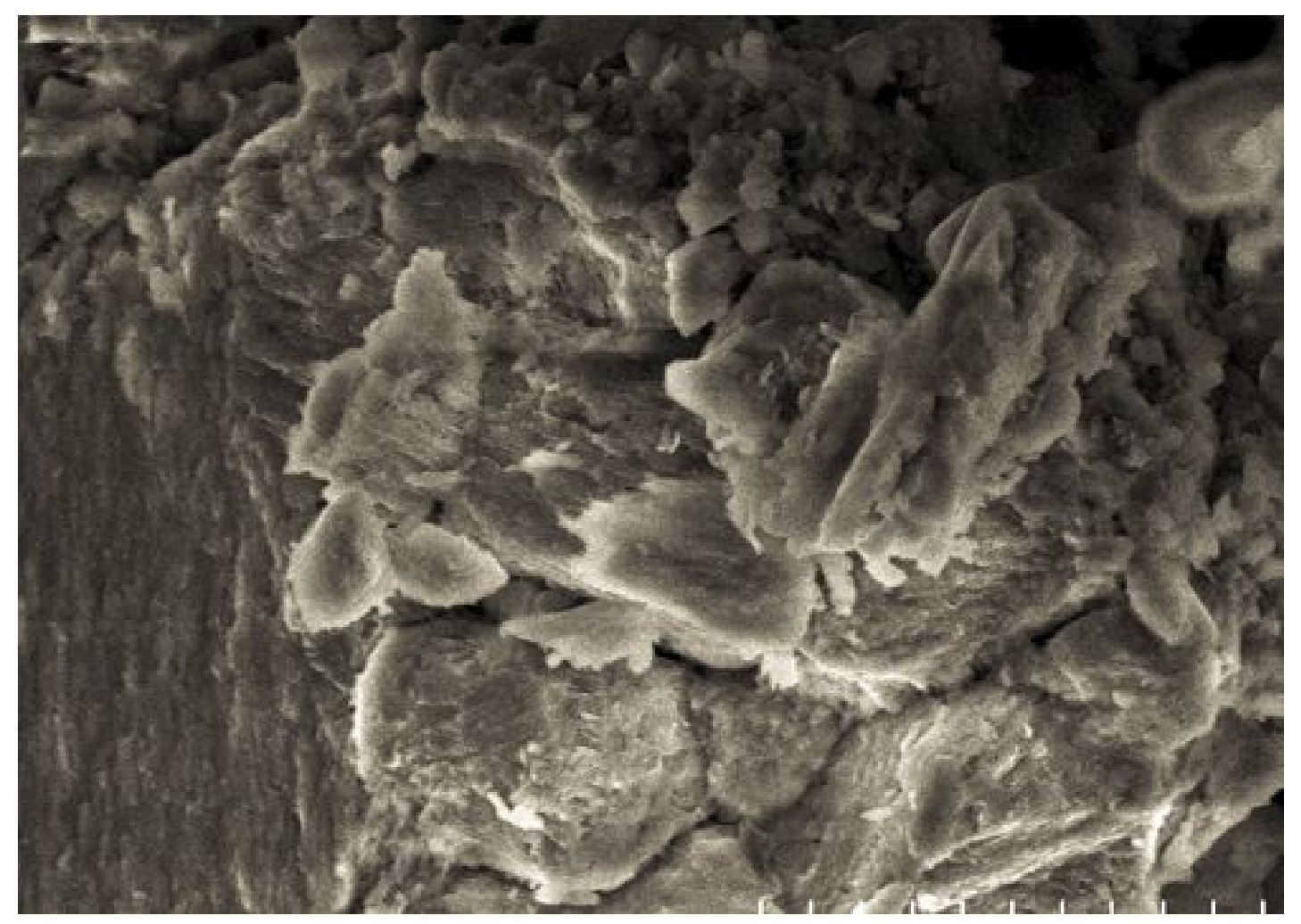

Figure S5. SEM image of colloidal $\mathrm{SiO}_{2} \mathrm{SM}(0.06)$ after TGA (scale bar $\left.=5 \mu \mathrm{m}\right)$.

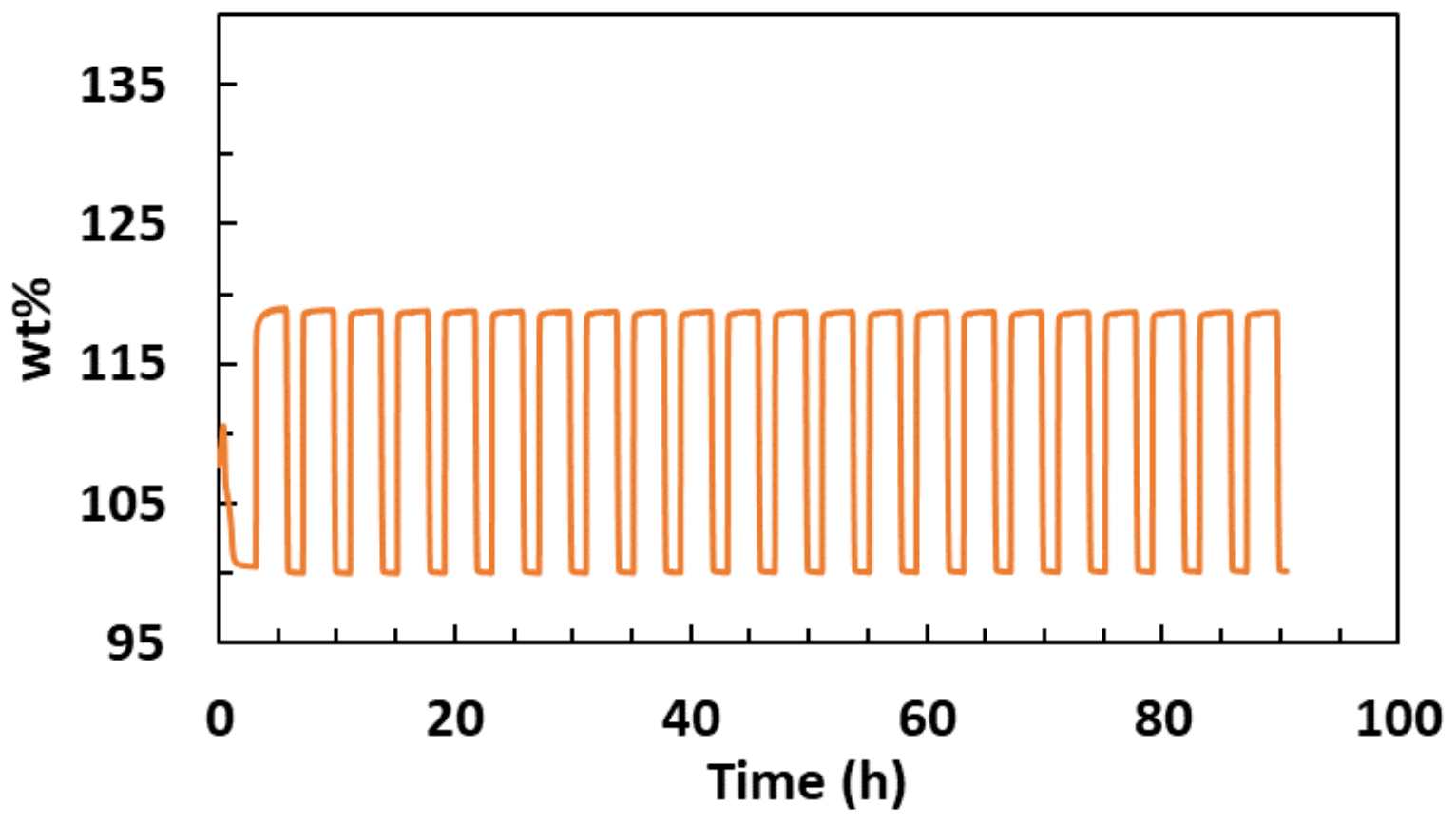

Figure S6. $2.5 \mathrm{~h} \mathrm{CO}_{2} /$ steam cycles under 50 vol\% $\mathrm{CO}_{2}$ at $923 \mathrm{~K}$ for XCit $\mathrm{SM}(0.12) \mathrm{E}$. 


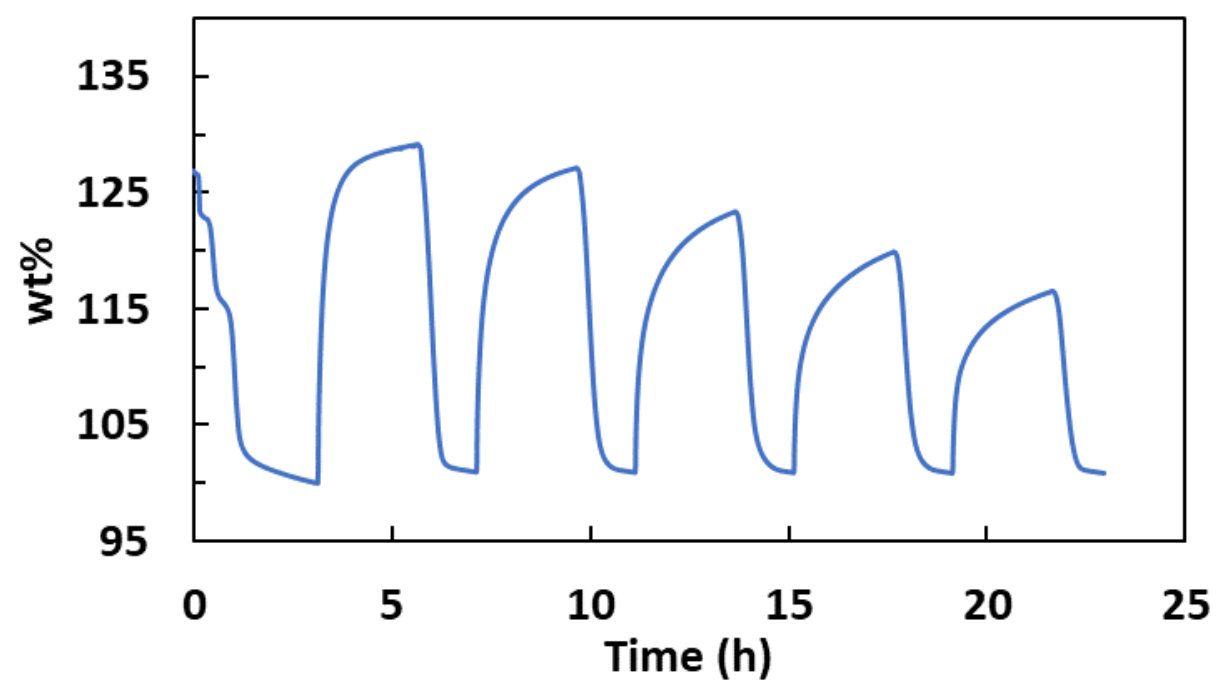

Figure S7. $2.5 \mathrm{~h} \mathrm{CO}_{2} /$ steam cycles under $50 \mathrm{vol} \% \mathrm{CO}_{2}$ at $923 \mathrm{~K}$ for $\mathrm{SM}(0.06) \mathrm{E}$

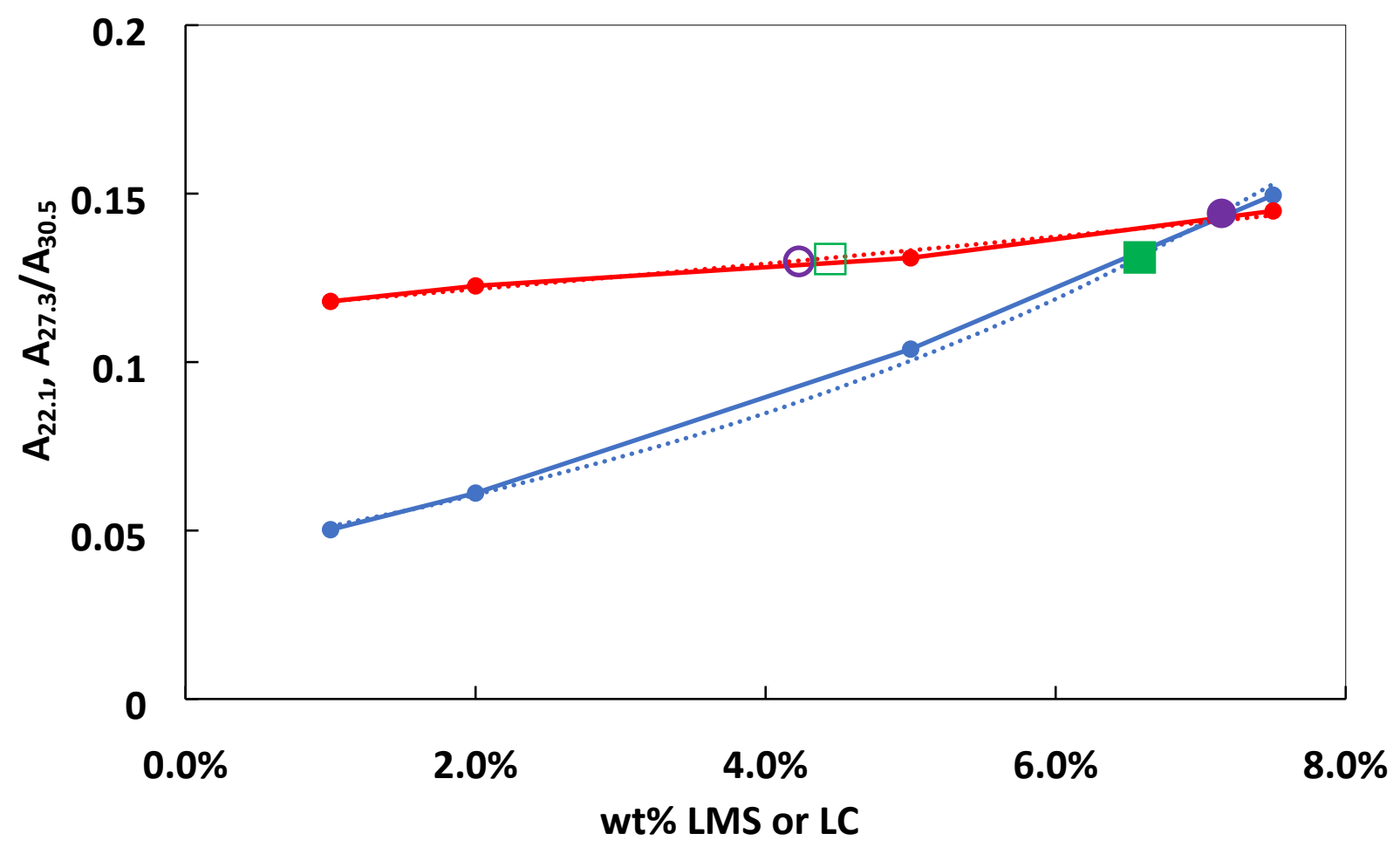

Figure S8. XRD calibration curve of LOS/LMS (red) and LOS/LC (blue) physical mixtures with $\mathrm{SM}(0.12)$ (purple circle) and $\mathrm{SM}(0.12) \mathrm{E}$ (green square) data points. 


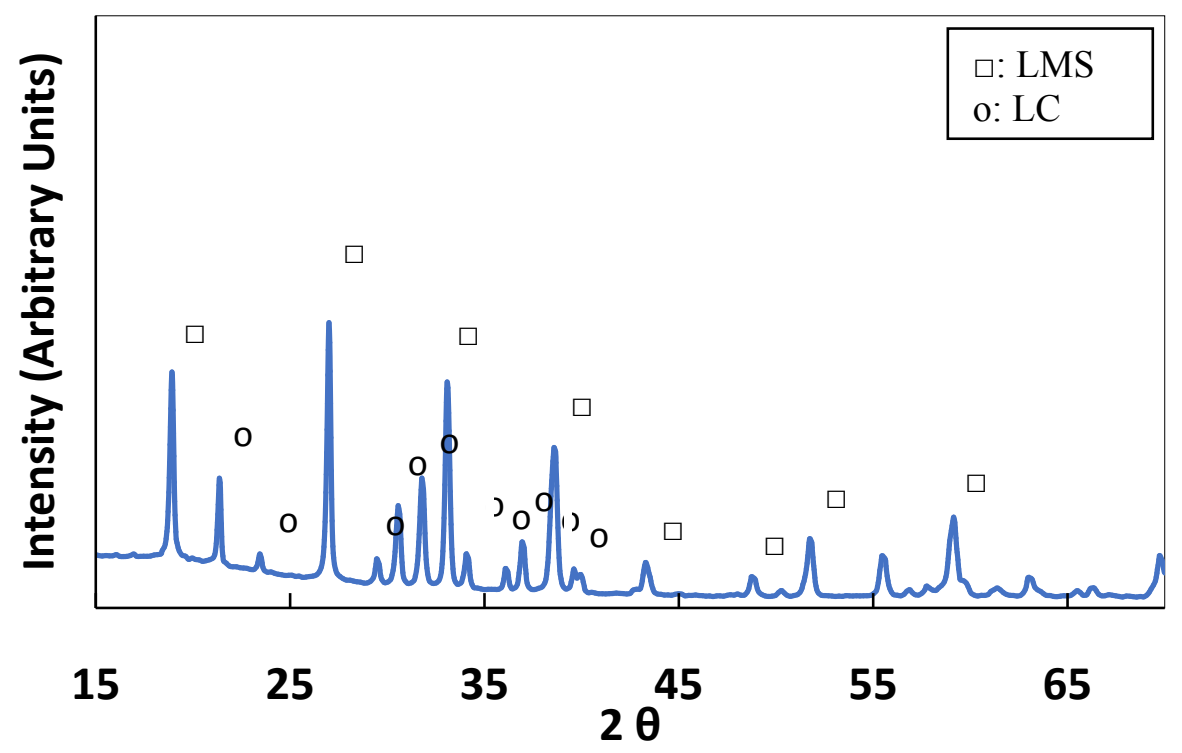

Figure S9. XRD pattern of carbonated $\mathrm{SM}(0.12)$ 